\title{
Intermittent versus daily dosing of meropenem in haemodialysis patients - A retrospective observational exploratory study
}

Vanda Ho ( $\square$ vanda_wt_ho@nuhs.edu.sg )

National University Hospital Singapore https://orcid.org/0000-0002-6413-4636

Felecia Tay

National University Singapore

Jia En Wu

National University Hospital Singapore

Lionel Lum

National University Hospital Singapore

Paul Tambyah

National University Hospital Singapore

Research article

Keywords:

Posted Date: April 30th, 2020

DOl: https://doi.org/10.21203/rs.3.rs-22000/v1

License: (c) (1) This work is licensed under a Creative Commons Attribution 4.0 International License. Read Full License 


\section{Abstract}

\section{Background}

Extended microbial resistance is very common in patients on haemodialysis, often needing long courses of carbapenems. This results in increased length of stay in hospital and its complications. However, carbapenems can be given intermittently and avoid this problem.

We aim to describe the clinical outcomes of intermittent versus daily meropenem in thrice weekly haemodialysed patients.

Methods: 125 records were examined retrospectively. Data collected includes patient demographics, clinical interventions such as source control, meropenem dose and regime, and clinical outcomes such as length of hospital stay (LOS), 30-day readmission rates and adverse events.

Results: Mean age was $62.6 \pm 1.3$ years, $56 \%$ were male. $65.6 \%$ were on haemodialysis secondary to diabetic nephropathy, and $84 \%$ were on thrice-weekly regime. Most common bacteria cultured was Klebsiella pneumoniae (14.4\%), the most common indication for meropenem was pneumonia (32\%) and mainly used for cure (65\%). Mean duration of therapy on meropenem was $11.5 \pm 1.2$ days, 8 patients needed more than 30 days of meropenem. $59.2 \%$ did not have intervention for source control.

$87.2 \%$ received daily dosing of meropenem. $6.4 \%$ patients received intermittent dosing of meropenem only, $4.8 \%$ patients received both types of dosing regimens. Dosing was variable across both arms.

LOS of the index admission was shorter for the intermittent arm, $15.5 \pm 2.7$ days compared to daily arm, $34.7 \pm 2.7$ days. Though 30 -day readmission was higher ( $50 \%$ in the intermittent arm versus $33.1 \%$ ), total LOS in the year was still lower ( $49 \pm 18.0$ days in the intermittent arm versus $53.3 \pm 14.9$ days). There were few adverse events in both arms.

Conclusion: Our study suggests that intermittent dosing of meropenem may be helpful for reducing hospital length of stay. We suggest for randomised controlled trials to prove its safety and efficacy.

\section{Background}

End-stage renal failure (ESRF) patients on haemodialysis often have multiple co-morbidities and are susceptible to bacterial infections(1) due to a variety of reasons related to the host(2) and surroundings(3). In many parts of the world, these infections are often caused by extended spectrum $\beta$ lactamase (ESBL) - producing gram-negative bacteria requiring antibiotics such as meropenem, which can be only administered intravenously(4).

Renally-adjusted meropenem in haemodialysis patients is administered once daily based on product labelling. Infections such as osteomyelitis or pyogenic abscesses require several weeks of treatment with antibiotics. This often results in prolonged hospitalisation, an admission to subacute hospital to 
complete the antibiotic course or the use of outpatient antibiotic therapy services. ESRF patients would receive meropenem post-dialysis on haemodialysis days and come to the hospital for administration of meropenem on non-haemodialysis days. However, intravenous access is required, usually via a peripherally-inserted central catheter (PICC). This may be a problem in haemodialysis patients who commonly have limited vascular access and are more susceptible to line-related sepsis. Furthermore, there is significant stress from logistics on the patient and their caregiver.

An alternative meropenem regime involving intermittent thrice-weekly dosing administered post-dialysis may be feasible. There are reports demonstrating its efficacy(5). Pharmacokinetic data suggest that reduced clearance in ESRF patients leads to longer half-life of meropenem and this may be sufficient to achieve effective concentrations with intermittent dosing(6). Practically there are many potential benefits. This regime may reduce hospital length of stay (LOS) and the need for PICC, thus reducing its associated costs and complications, such as nosocomial infections. It also circumvents the logistical issues of daily administration at a healthcare institution. The evidence for toxicity with high dose intermittent meropenem is not clear $(7,8)$, though meropenem and its metabolite have also been shown to be effectively removed by haemodialysis(6). Unfortunately, intermittent meropenem dosing regimens for haemodialysis patients have not been widely used due to limited published data.

To investigate the feasibility of intermittent meropenem dosing regime in ESRF patients, we describe clinical outcomes of intermittent versus daily meropenem in stable thrice-weekly haemodialysed patients in terms of length of hospital stay and 30-day readmission rates.

\section{Methods}

A single-centre retrospective cohort study was conducted in the National University Hospital (NUH), Singapore. Data was obtained from the NUH's Antimicrobial Stewardship Programme database from May 2015 to February 2017. Inclusion criteria were adult patients ( $\geq 21$ years old) on stable intermittent haemodialysis who had received at least 3 days of meropenem. Our study was approved by the local ethics board (domain specific review board DSRB approval number: 2018/00578). 125 patient records were examined. Data collection categories were patient demographics, clinical data, meropenem regime and clinical outcomes, as detailed in Appendix 1. Demographic data included age, sex, ethnicity and Charlson Comorbidity Index (CCl)(9). Involvement of the infectious disease team was documented. Indication for haemodialysis (e.g. diabetic nephropathy) and the haemodialysis regime (i.e. intermittent haemodialysis thrice-weekly, sustained low efficiency dialysis (SLED), continuous renal replacement therapy (CRRT)) were recorded. Patients were considered immunosuppressed if they had an active malignancy, on active chemotherapy, on high dose of steroids or other immunosuppressants. Clinical data included site of infection, microbiological cultures (both poly-microbial and monomicrobial infections), antimicrobial susceptibilities, and interventions performed for source control. The aim of administering meropenem (i.e. cure - culture-guided short-term course seeking to eradicate the infection 
versus suppression - culture-guided long term course to reduce bacterial load), dosing regimen (i.e. intermittent, daily or both) and duration were also included. Meropenem $500 \mathrm{mg}$ was given as a bolus; $1000 \mathrm{mg}, 2000 \mathrm{mg}$ and $3000 \mathrm{mg}$ were given as an hour-long infusion. Clinical outcomes included LOS during the index admission, the intensive care unit (ICU) LOS (if any), 30-day readmission rate, number of relapses with the same microorganism within one year, total LOS within the year for the same infection, adverse events specific to meropenem and mortality within one year. As this study was done retrospectively and group sizes are different, descriptive analyses were used. No statistical comparisons were carried out. Results are reported to 1 decimal place.

\section{Results}

125 patient records were examined.

\section{Demographics}

Mean age $62.6 \pm 1.3$ years, of which $56.0 \%$ were male $(n=70)$. The ethnic distribution was comparable to the Singapore population distribution. $79.2 \%$ were from government subsidised wards $(n=99)$. $54.4 \%$ were admitted under nephrology ( $n=68)$, followed by surgery $(n=29,23.2 \%) .49(39.8 \%)$ were referred to the infectious disease team.

$72.8 \%$ were not immunocompromised ( $\mathrm{n}=91) .21$ (16.8\%) had active malignancy, $8(6.4 \%)$ on chemotherapy and $14(11.2 \%)$ were on long term steroids.

Majority were on dialysis due to diabetic renal disease $(n=82,65.6 \%)$, followed by glomerulonephritis $(n=10,8.0 \%)$, hypertensive renal disease $(n=9,7.2 \%)$ and sepsis-induced renal failure $(n=4,3.2 \%) .84 .0 \%$ $(n=104)$ had dialysis thrice-weekly, with $3(2.4 \%)$ requiring CRRT and $8(6.4 \%)$ requiring SLED. 4 patients (3.2\%) were on peritoneal dialysis on admission and converted to haemodialysis during their inpatient stay.

\section{Microbiological Results}

Samples obtained were mainly from blood $(n=73,58.4 \%)$. About a quarter $(n=34,27.2 \%)$ were from wound swabs, a minority ( $n=19,15.2 \%)$ were from urine cultures. About half $(n=65,52.0 \%)$ did not have any positive microbiological result. The most common bacteria cultured was Klebsiella pneumoniae $(n=18,14.4 \%)$, followed by Escherichia coli $(n=14,11.2 \%)$ Staphylococcus aureus $(n=11,8.8 \%)$, Pseudomonas aeruginosa $(n=10,8.0 \%)$, Enterobacter cloacae $(n=7,5.6 \%)$ and Streptococcus pneumoniae $(\mathrm{n}=4,3.2 \%)$. When cultured, bacteria were sensitive to meropenem by EUCAST (European Committee on Antimicrobial Susceptibility Testing) criteria.

\section{Clinical Interventions}

Most common diagnosis was pneumonia $(n=40,32 \%)$, followed by skin and soft tissue infections $(n=22$, $17.6 \%)$. 
Meropenem was largely used with a culture-guided curative intent, $(n=78,65 \%)$. Indications include bone and joint infections $(n=5,6.4 \%)$, bacteraemia $(n=9,11.5 \%)$, chest infection $(n=23,29.5 \%)$, intra-abdominal infection $(n=4,5.1 \%)$, line sepsis $(n=2,2.6 \%)$, skin and soft tissue infection $(n=15,19.2 \%)$, urinary tract infection $(n=9,11.5 \%)$ and sepsis with no source $(n=11,14.1 \%)$. In contrast, meropenem was used for suppression of chronic infections in $9(7.9 \%)$ patients. Indications include bone and joint infections $(\mathrm{n}=2$, $22.2 \%)$, bacteraemia $(n=1,1115 \%)$, intra-abdominal infection $(n=1,11.1 \%)$, skin and soft tissue infection $(n=4,44.4 \%)$, and sepsis with no source $(n=1,11.1 \%) .2$ were on meropenem due to hypersensitivity to other narrow spectrum antibiotics. Mean duration of therapy on meropenem was $11.5 \pm 1.2$ days, with 8 patients requiring more than 30 days of meropenem. The longest duration was a total of 109 days.

$87.2 \%$ received daily dosing of meropenem $(n=109,87.2 \%) .8(6.4 \%)$ patients received intermittent dosing of meropenem only, and $6(4.8 \%)$ patients received both types of dosing regimens. All patients on intermittent dosing were on a thrice-weekly dosing regime. Dosing of meropenem varied, with 8 (57.1\%) patients on $2 \mathrm{~g} / 2 \mathrm{~g} / 3 \mathrm{~g}$ dosing, $3(21.4 \%)$ on $2 \mathrm{~g} / 2 \mathrm{~g} / 2 \mathrm{~g}, 2$ (14.3\%) on $1 \mathrm{~g} / 1 \mathrm{~g} / 1 \mathrm{~g}$ and $1(7.1 \%)$ on $1 \mathrm{~g} / 1 \mathrm{~g} / 2 \mathrm{~g}$. This variation of dosing was also seen in the daily dosing regime, with a range from $500 \mathrm{mg}$ once to twice daily, to $1 \mathrm{~g}$ once to thrice daily. Comparison of variables amongst different meropenem dosing regime is described in Table 1.

$59.2 \%$ did not have any intervention for source control $(n=74)$. Indications for meropenem include bone and joint infections $(n=1,1.4 \%)$, bacteraemia $(n=6,8.1 \%)$, chest infection $(n=32,43.2 \%)$, intra-abdominal infection $(n=3,4.1 \%)$, line sepsis $(n=2,2.7 \%)$, skin and soft tissue infection $(n=4,5.4 \%)$, urinary tract infection $(n=8,10.8 \%)$ and sepsis with no source $(n=18,24.3 \%)$. Of those who received interventions, 9 (7.2\%) had percutaneous drainage, $22(17.6 \%)$ major surgery and $15(12.0 \%)$ had minor surgical procedures. For those who received percutaneous drainage, indications for meropenem include chest infection $(n=5,55.6 \%)$, intra-abdominal infection $(n=3,33.3 \%)$ and skin and soft tissue infection $(n=1$, $11.1 \%)$. For those who received major surgery, indications for meropenem include bone and joint infections $(n=7,31.8 \%)$, bacteraemia $(n=2,9.1 \%)$, chest infection $(n=1,4.6 \%)$, intra-abdominal infection $(n=1,4.6 \%)$, skin and soft tissue infection $(n=10,45.5 \%)$ and sepsis with no source $(n=1,4.6 \%)$. For those who had minor surgical procedures, indications for meropenem include bone and joint infections $(n=5$, $33.3 \%)$, chest infection $(n=1,6.7 \%)$, intra-abdominal infection $(n=1,6.7 \%)$, skin and soft tissue infection $(n=8,53.3 \%)$.

\section{Clinical Outcomes}

Mean LOS in hospital was $36.6 \pm 3.6$ days. $38(69.6 \%)$ required ICU admission with a mean ICU LOS of $10.2 \pm 2.0$ days. LOS was longer in the group with continuous meropenem ( $34.7 \pm 2.7$ days verses $15.5 \pm 2.7$ days), though antibiotic duration was shorter ( $8.7 \pm 0.6$ days versus $23.4 \pm 6.2$ days). This longer duration of antibiotics despite shorter LOS is possible as patients on intermittent meropenem dosing receive their doses outpatient at their dialysis centres.

94.4\% ( $\mathrm{n}=118)$ did not have any documented adverse events. 5 patients developed Clostridium difficile colitis, 1 developed allergic reactions and 1 acquired Carbapenem-resistant Enterobacteriaceae (CRE) 
positive on screening. $43(34.4 \%)$ were readmitted within 30 days. $28(22.4 \%)$ had relapses within the year. Mean total LOS within the year was $64.8 \pm 15.0$ days. 35 (28\%) died within 1 year of discharge. Of the 20 patients with known cause of death, 7 died from cardiac-related causes, 3 from ESRF and 10 from infection-related causes, mostly secondary to pneumonia.

\section{Discussion}

To our knowledge, this is the first study to describe clinical outcomes of intermittent versus daily dosing of meropenem in patients on a stable haemodialysis regime. There is evidence that intermittent dosing of beta lactams is safe and effective for severe infections(10). Whilst intermittent dosing in patients on haemodialysis has been used with other beta-lactam antibiotics such as cefepime(11) and cefazolin(12), this approach has not been as widely adopted with carbapenems despite some pharmacokinetic and pharmacodynamic data $(13,14)$.

There were few adverse events in patients treated with both intermittent and daily meropenem, in line with previous reports on the tolerability of meropenem(15).

Our data reveals that there is much variation in meropenem dosing for both daily and intermittent dosing. This lack of standardisation highlights the need for interventional trials to clearly determine the optimal dosing regimen.

In our small cohort, intermittent dosing appears to be a feasible approach to reduce healthcare utilisation, with comparable clinical efficacy to the more widely-used daily dosing. The trend where patients on intermittent dosing had reduced hospital stay without major adverse events is encouraging.

There is much impetus to adopt intermittent dosing of meropenem in ESRF patients on haemodialysis. Patients on haemodialysis tend towards developing depression and poor quality of life(16), which have been found to correlate with longer length of stay in hospital $(17,18)$. An approach which reduces hospital stay and allows the patient to resume normal activities is likely to be welcomed. This is especially so in the era of escalating healthcare costs which is a major concern for haemodialysis patients(19).

\section{Strengths and Limitations}

We believe that our small study would help pave the way forward towards use of intermittent dosing in our haemodialysis population, with potential to reduce hospital costs and improve the quality of life of our patients.

As this is an exploratory retrospective study, our sample size is small and dosing arms were unequally distributed. Hence, our study is limited to being descriptive with minimal statistical analysis. We were unable to conclude non inferiority in both approaches. To increase sample size, we included a small number of patients on renal replacement therapy secondary to sepsis-induced renal failure. We also included patients on continuous renal replacement therapy and sustained low efficiency dialysis. While 
this is not directly comparable to chronic end-stage renal disease on stable intermittent haemodialysis, this exploratory study helps to tailor the study population for further trials.

\section{Conclusions}

Our study suggests that intermittent dosing of meropenem may be helpful for reducing hospital days. We would suggest for randomised controlled trials to prove its safety and efficacy.

\section{Abbreviations}

CCI - Charlson Comorbidity Index CRE - Carbapenem-resistant Enterobacteriaceae CRRT - continuous renal replacement therapy ESRF - end-stage renal failure ESBL - extended spectrum $\beta$-lactamase EUCAST - European Committee on Antimicrobial Susceptibility Testing ICU - intensive care unit LOS - length of stay NUH - National University Hospital PICC - peripherally-inserted central catheter SLED - sustained low efficiency dialysis

\section{Declarations}

Ethics Approval and consent to participate: Domain Specific Review Board under National Healthcare Group, reference number 2018/00578

Consent for publication: No consent needed from participants as data are all anonymised

Availability of data and material: NA

Competing interests: None

Funding: None

Author's Contributions: VH, FT, JEW, LL and PT jointly conceptualised and prepared the manuscript. All authors have read and approved the manuscript.

Acknowledgements: None

\section{References}

1. Bae EH, Kim HY, Kang YU, Kim CS, Ma SK, Kim SW. Risk factors for in-hospital mortality in patients starting hemodialysis. Kidney Research and Clinical Practice. 2015 Sep 1;34(3):154-9.

2. Chonchol M. Neutrophil dysfunction and infection risk in end-stage renal disease. Seminars in dialysis.;19(4):291-6. 
3. Pop-Vicas A, Strom J, Stanley K, D’Agata EMC. Multidrug-resistant gram-negative bacteria among patients who require chronic hemodialysis. Clinical journal of the American Society of Nephrology: CJASN. 2008 May;3(3):752-8.

4. Saito T, Sawazaki R, Ujiie K, Oda M, Saitoh H. Possible Factors Involved in Oral Inactivity of Meropenem, a Carbapenem Antibiotic. Pharmacology Pharmacy. 2012;03(02):201-6.

5. Chimata M, Nagase M, Suzuki Y, Shimomura M, Kakuta S. Pharmacokinetics of meropenem in patients with various degrees of renal function, including patients with end-stage renal disease. Antimicrob Agents Chemother. 1993 Feb;37(2):229-33.

6. Leroy A, Fillastre JP, Borsa-Lebas F, Etienne I, Humbert G. Pharmacokinetics of meropenem (ICI $194,660)$ and its metabolite (ICI 213,689) in healthy subjects and in patients with renal impairment. Antimicrobial agents and chemotherapy. 1992 Dec;36(12):2794-8.

7. Imani S, Buscher H, Marriott D, Gentili S, Sandaradura I. Too much of a good thing: A retrospective study of $\beta$-lactam concentration-toxicity relationships. Journal of Antimicrobial Chemotherapy. 2017 Oct 1;72(10):2891-7.

8. Cojutti P, Sartor A, Righi E, Scarparo C, Bassetti M, Pea F. Population pharmacokinetics of high-dose continuous-infusion meropenem and considerations for use in the treatment of infections due to KPC-producing Klebsiella pneumoniae. Antimicrobial Agents and Chemotherapy. 2017;61(10).

9. Charlson ME, Pompei P, Ales KL, MacKenzie CR. A new method of classifying prognostic comorbidity in longitudinal studies: Development and validation. Journal of Chronic Diseases. 1987;40(5):37383.

10. Meyer B, Guttmann C, Dittrich E, Schmaldienst S, Thalhammer F. Intermittent administration of betalactam-antibiotics for treatment of severe infection in hemodialysis patients. European journal of medical research. 2005 Apr 20;10(4):140-4.

11. Schmaldienst S, Traunmüller F, Burgmann H, Rosenkranz AR, Thalhammer-Scherrer R, Hörl WH, et al. Multiple-dose pharmacokinetics of cefepime in long-term hemodialysis with high-flux membranes. Eur J Clin Pharmacol. 2000 Apr;56(1):61-4.

12. Kuypers D, Vanwalleghem J, Maes B, Messiaen T, Vanrenterghem $Y$, Peetermans WE. Cefazolin serum concentrations with fixed intravenous dosing in patients on chronic hemodialysis treatment. Nephrology, dialysis, transplantation: official publication of the European Dialysis and Transplant Association - European Renal Association. 1999 Aug;14(8):2050-1.

13. Thalhammer F, Hörl WH. Pharmacokinetics of meropenem in patients with renal failure and patients receiving renal replacement therapy. Clin Pharmacokinet. 2000 Oct;39(4):271-9.

14. Cunha BA. Meropenem in elderly and renally impaired patients. Vol. 11, International Journal of Antimicrobial Agents. Elsevier; 1999. p. 167-77.

15. Norrby SR, Gildon KM. Safety profile of meropenem: a review of nearly 5,000 patients treated with meropenem. Scand J Infect Dis. 1999;31(1):3-10.

16. Teles F, Amorim de Albuquerque AL, Freitas Guedes Lins IK, Carvalho Medrado P. Falcão Pedrosa Costa A. Quality of life and depression in haemodialysis patients. Psychology health medicine. 
2018;23(9):1069-78.

17. Lacson E, Bruce L, Li NC, Mooney A, Maddux FW. Depressive affect and hospitalization risk in incident hemodialysis patients. Clin J Am Soc Nephrol. 2014;9(10):1713-9.

18. Chan L, Tummalapalli SL, Ferrandino R, Poojary P, Saha A, Chauhan K, et al. The Effect of Depression in Chronic Hemodialysis Patients on Inpatient Hospitalization Outcomes. Blood Purif. 2017 Mar;43(1-3)(1):226-34.

19. Kshirsagar AV, Hogan SL, Mandelkehr L, Falk RJ. Length of stay and costs for hospitalized hemodialysis patients: nephrologists versus internists. Journal of the American Society of Nephrology: JASN. 2000 Aug;11(8):1526-33.

\section{Tables}

Table 1. Comparison of different meropenem dosing regimens 


\begin{tabular}{|c|c|c|c|}
\hline & Daily $(\mathrm{n}=109,87.2 \%)$ & $\begin{array}{l}\text { Intermittent }(\mathrm{n}=8 \text {, } \\
6.4 \%)\end{array}$ & Both $(\mathrm{n}=6,4.8 \%)$ \\
\hline \multicolumn{4}{|l|}{ Demographics } \\
\hline Age in years (mean $\pm \mathrm{SD}$ ) & $62.8 \pm 1.4$ & $57.8 \pm 2.8$ & $63.2 \pm 5.0$ \\
\hline Males (n, \%) & $59(54.1)$ & $4(50.0)$ & $5(83.3)$ \\
\hline Charlsons Comorbidity Index (mean \pm SD) & $7.9 \pm 0.3$ & $8.4 \pm 0.6$ & $8.0 \pm 0.9$ \\
\hline Immunocompromised(n, \%) & $29(26.6)$ & $2(25.0)$ & $1(16.7)$ \\
\hline Haemodialysis indication due to diabetes (n, \%) & $70(64.2)$ & 7 (87.5) & $5(83.3)$ \\
\hline Haemodialysis regime: 3x/week (n, \%) & $91(83.5)$ & $8(100.0)$ & $6(100.0)$ \\
\hline \multicolumn{4}{|l|}{$\begin{array}{l}\text { Discipline of admission (n, \%) } \\
\text { Renal }\end{array}$} \\
\hline Referral to infectious disease team (n, \%) & $40(36.7)$ & $5(62.5)$ & $4(66.7)$ \\
\hline \multicolumn{4}{|l|}{ Clinical Interventions } \\
\hline Intervention performed for source control (n, \%) & 37 (33.9) & $2(25.0)$ & 0 \\
\hline $\begin{array}{l}\text { Purpose of meropenem (n, \%) } \\
\text { Cure } \\
\text { Suppression } \\
\text { Empirical }\end{array}$ & $\begin{array}{l}68(63.6) \\
7(6.5) \\
32(29.9)\end{array}$ & $\begin{array}{l}5(62.5) \\
2(25.0) \\
1(12.5)\end{array}$ & $\begin{array}{l}5(100.0) \\
0 \\
0\end{array}$ \\
\hline Duration of meropenem, days (mean \pm SD) & $8.7 \pm 0.6$ & $23.4 \pm 6.2$ & $47.8 \pm 14.1$ \\
\hline \multicolumn{4}{|l|}{ Clinical outcomes } \\
\hline LOS of index admission, days (mean \pm SD) & $34.7 \pm 2.7$ & $15.5 \pm 2.7$ & $109.7 \pm 48.8$ \\
\hline ICU length of stay, days (mean \pm SD) & $3.6 \pm 0.8$ & 0 & 0 \\
\hline Adverse events (n, \%) & $\begin{array}{l}5 \text { (4.6) } \\
\text { Clostridium difficilecolitis } \\
(\mathrm{n}=3) \\
\text { CRE colonisation }(\mathrm{n}=1) \\
\text { Drug hypersensitivity } \\
(\mathrm{n}=1)\end{array}$ & 0 & $\begin{array}{l}2(33.3) \\
\text { Clostridium difficile colitis } \\
(\mathrm{n}=2)\end{array}$ \\
\hline $\begin{array}{l}\text { 30-day readmissions(n, \%) } \\
1 \\
2\end{array}$ & $\begin{array}{l}32(29.4) \\
4(3.7) \\
\end{array}$ & $4(50.0)$ & $3(50.0)$ \\
\hline $\begin{array}{l}\text { Relapses within one year }(\mathrm{n}, \%) \\
1\end{array}$ & & & \\
\hline 2 & $14(13.1)$ & $2(25.0)$ & $2(33.3)$ \\
\hline 3 & $5(4.7)$ & $1(12.5)$ & 0 \\
\hline 4 & $1(0.9)$ & 1 (12.5) & 0 \\
\hline 5 & $\begin{array}{l}0 \\
1(0.9)\end{array}$ & $\begin{array}{l}0 \\
0\end{array}$ & $\begin{array}{l}0 \\
0\end{array}$ \\
\hline
\end{tabular}




\begin{tabular}{|l|l|l|l|}
$\begin{array}{l}\text { Total LOS within the year for the same infection, days } \\
(\text { mean } \pm \text { SD })\end{array}$ & $53.3 \pm 14.9$ & $49 \pm 18.0$ & $235 \pm 55$ \\
\hline Mortality within 1 year $(\mathrm{n}, \%)$ & $41(37.6)$ & $3(37.5)$ & $2(33.3)$ \\
\hline
\end{tabular}

\section{Appendix}

\section{APPENDIX 1: STUDY PRO-FORMA}

Patient No:

\section{SECTION 1: PATIENT INFORMATION}
Age (as of D1
meropenem):
Height Weight
(cm): $\quad(\mathrm{kg})$ :
Sex: Class: Ethnicity:
M/F A/B1/ Chinese/ Malay/Indian/
B2/ C Others (specify)

Charlsons Comorbidity Index score:

$\mathrm{PMH}$ (circle where appropriate):

DM, HTN, Hyperlipidemia, IHD, CVA, PVD
Discipline of admission (circle where appropriate):

Renal/ RCCM/ Cardio/ Endocrine/ Gastro/ Neuro/ Rheum/ GRM/ Gen Med

ID referral: $\mathrm{Y} / \mathrm{N}$

Any immunocompromised state(s):

Chemotherapy, TNF blockers, steroids, DXT, others please specify:

Haemodialysis regime (circle where appropriate):

Indication for HD:

DM, HTN, ADPKD, GN, AIN, sepsis

3x/week 4x/week Daily

Others - please specify:

SLED

\section{SECTION 2: INFECTION}


Anatomical site of infection:

Lung/ skin and soft tissue/ bones and joints/ intra-abdominal/ urinary tract/ blood/ lines/ nil source
Initial microbiological cultures result and sensitivities:

Histopathological results (if any)
Intervention for source control:

$\square$ Percutaneous drainage $\square$ Surgery $\square$ N/A
Purpose of meropenem treatment:

$\square$ Cure $\square$ Suppression

Details:

Meropenem treatment:

Start date:

Stop date:

Total number of days on meropenem:

Regime: daily intermittent

If intermittent

- Frequency: 3x/ week others, please specify:

- $\quad$ Dosing (g)

\section{SECTION 3: CLINICAL OUTCOMES}

Adverse events from meropenem (circle where appropriate):

- Seizure/ anaphylaxis/ allergy

Total length of hospital stay (days):

Number of readmissions in 30 days: 
- Reason for admission:

Number of clinical relapses within 1 year:

- If yes, total length of hospital stay for the same infection in 1 year:

Mortality within 1 year: $\mathrm{Y} / \mathrm{N}$

- If yes, CCOD: 